\title{
Solutions analytiques de problèmes de contact en grandes déformations
}

\author{
Éric Chamberland ${ }^{1, a}$, André Fortin ${ }^{1}$ et Nicolas Tardieu ${ }^{2}$ \\ 1 GIREF, Université Laval Pavillon Vachon, 1045 avenue de la médecine, Québec, G0V 0A6, Canada \\ 21 Av. Général de Gaulle, 91128 Clamart Cedex, France
}

Reçu le 8 juillet 2010, accepté le 12 juillet 2010

\begin{abstract}
Résumé - Il est impératif pour l'ingénieur de s'assurer de la validité de ses outils de modélisation numérique et il lui revient de vérifier s'ils donnent la solution attendue pour un problème donné. Dans un logiciel d'éléments-finis, ceci peut être fait par l'utilisation de solutions analytiques lorsqu'elles existent, ou par la méthode des solutions manufacturées (MSM). Nous proposons dans ce travail d'étendre la MSM aux problèmes de contact unilatéral en grandes déformations pour lesquels, à notre connaissance, il n'existe pas de solutions analytiques non triviales. Nous montrerons comment l'utiliser comme un outil de vérification pour l'ingénieur. De plus, nous analyserons la précision et les taux de convergence de différents espaces d'approximation mixtes (déplacement-pression de contact), sur des éléments triangulaires et quadrangulaires.
\end{abstract}

Mots clés : Solutions manufacturées / solutions analytiques / contact unilatéral / grandes déformations / éléments-finis quadratiques

\begin{abstract}
Analytical solutions to large deformation contact problems. It is essential for an engineer to verify the exactitude of the numerical tools he is using, as it is his duty to ensure that they give the expected solution to a given problem. In a finite-element code, this can be achieved by using analytical solutions when they exist, or by the method of manufactured solutions (MMS). In this work, we propose to extend the MMS to large deformation unilateral contact problems for which, to our knowledge, there exists no simple analytical solutions. We will show how this method can be a very efficient verification tool for the engineer. We will also study the precision and convergence order of different choices of mixed spaces (displacement-contact pressure), on triangular and quadrangular elements.
\end{abstract}

Key words: Manufactured solutions / analytical solutions / unilateral contact / large deformations / quadratic finite-elements

\section{Sur la vérification de logiciels}

La vérification de logiciel est un aspect très important du génie logiciel. On y inclut les tests de nonrégression, où l'on compare les résultats d'un calcul dans l'état actuel du logiciel avec des résultats obtenus et vérifiés antérieurement, ainsi que les compilations multiarchitectures, multi-compilateurs effectuées sur une base régulière. Ce premier type de vérification assure un niveau minimal de qualité du logiciel : aussi longtemps que les résultats des tests ne changent pas, le logiciel

\footnotetext{
a Auteur pour correspondance :

eric.chamberland@giref.ulaval.ca
}

maintient le niveau de qualité qu'il avait lors de la production des résultats étalons. L'ensemble de ces vérifications est présenté comme faisant partie de l'assurance qualité logiciel par Roy [1].

Deux types de problèmes peuvent toutefois persister malgré ces tests quotidiens. Il se peut en effet que certaines parties du logiciel ne soient pas couvertes par ces tests. Il s'agit alors d'une partie du logiciel qui n'est pas vérifiée de façon régulière et qui peut subitement donner de faux résultats. Pire encore, et c'est le deuxième type de problèmes, il se peut qu'elle n'ait jamais donné de bons résultats. En modélisation numérique, et plus spécifiquement en éléments-finis, les problèmes possèdent rarement des solutions analytiques connues. Comment 


\section{Nomenclature}

\begin{tabular}{|c|c|}
\hline $\mathrm{d} A:$ & unité d'aire à la frontière du domaine non déformé \\
\hline $\mathrm{d} V:$ & unité de volume du domaine non déformé \\
\hline$E_{\mathrm{Y}}:$ & module de Young \\
\hline $\boldsymbol{F}:$ & gradient de déformation \\
\hline$g:$ & distance orientée donnée par $\left(\boldsymbol{x}_{1}-\boldsymbol{x}_{2}\right) \cdot \boldsymbol{n}_{2}$ \\
\hline $\boldsymbol{h}_{0}:$ & condition aux limites de Neumann appliquée à la frontière $\Gamma_{\mathrm{N}}^{0}$ \\
\hline$J_{\mathrm{s}}:$ & rapport entre les aires déformée et non déformée $(\mathrm{d} a / \mathrm{d} A)$ \\
\hline$k_{0}:$ & condition aux limites de contact appliquée sur la frontière $\Gamma_{\mathrm{C}}^{0}$ \\
\hline$N:$ & normale à la frontière du domaine $\Omega_{1}^{0}$ \\
\hline$n:$ & normale à la frontière du domaine $\Omega_{1}^{t}$ \\
\hline$n_{2}:$ & normale à la frontière du domaine $\Omega_{2}$ au point $\boldsymbol{x}_{2}$ \\
\hline$r_{0}:$ & expression du terme source dans le domaine $\Omega_{1}^{0}$ \\
\hline$S:$ & second tenseur de Piola-Kirchoff \\
\hline$u:$ & déplacement \\
\hline $\boldsymbol{u}_{h}:$ & déplacement discret \\
\hline$x_{1}:$ & position d'un point du domaine $\Omega_{1}^{t}: \boldsymbol{x}_{1}=\boldsymbol{X}_{1}+\boldsymbol{u}$ \\
\hline$x_{2}:$ & position résultant de la projection du point $\boldsymbol{x}_{1}$ de $\Gamma_{\mathrm{C}}^{t}$ sur la frontière de $\Omega_{2}$ \\
\hline $\boldsymbol{X}_{1}:$ & position d'un point du domaine $\Omega_{1}^{0}$ \\
\hline$\Gamma_{\mathrm{D}}^{0}:$ & partie de la frontière de $\Omega_{1}^{0}$ où on impose des conditions de Dirichlet \\
\hline$\Gamma_{\mathrm{N}}^{0}:$ & partie de la frontière de $\Omega_{1}^{0}$ où on impose des conditions de Neumann \\
\hline$\Gamma_{\mathrm{C}}^{0}:$ & partie de la frontière de $\Omega_{1}^{0}$ éligible au contact \\
\hline$\Gamma_{C}^{\mathrm{t}}:$ & partie de la frontière de $\Omega_{1}^{t}$ éligible au contact \\
\hline$\lambda:$ & pression de contact \\
\hline$\lambda_{h}:$ & pression de contact discrète \\
\hline$\nabla_{X}:$ & gradient calculé dans la configuration non déformée \\
\hline$\nu:$ & coefficient de Poisson \\
\hline$\Omega_{1}^{0}:$ & domaine de calcul dans la configuration non déformée \\
\hline$\Omega_{1}^{t}:$ & domaine de calcul dans la configuration déformée au temps $t$ \\
\hline$\Omega_{2}:$ & obstacle rigide \\
\hline$\phi_{u}:$ & fonctions tests de $\boldsymbol{u}_{h}$ \\
\hline$\phi_{\lambda}:$ & fonctions tests de $\lambda_{h}$ \\
\hline$\Pi:$ & premier tenseur de Piola-Kirchoff \\
\hline$\sigma:$ & contraintes de Cauchy \\
\hline$\sigma_{\mathrm{nn}}:$ & partie normale des contraintes de Cauchy \\
\hline$\sigma_{\mathrm{nn}}^{+}:$ & partie normale et positive des contraintes de Cauchy $\left.\left.\left(\sigma_{\mathrm{nn}}^{+} \in\right] 0, \infty\right]\right)$ \\
\hline$\sigma_{\mathrm{nn}}^{-}:$ & partie normale et négative des contraintes de Cauchy $\left(\sigma_{\mathrm{nn}}^{-} \in[-\infty, 0[)\right.$ \\
\hline$\sigma_{\mathrm{t}}:$ & partie tangentielle des contraintes de Cauchy \\
\hline
\end{tabular}

peut-on alors valider des résultats pour lesquels il n'existe aucune solution analytique connue?

La MSM, introduite par Steinberg et Roache [2], est utilisée depuis longtemps en mécanique des fluides (voir Pelletier et al. [3]). Salari et Knupp [4] présentent un rapport dans lequel plusieurs aspects de la vérification de code sont abordés. On y retrouve la MSM avec plusieurs détails intéressants, dont une série de 21 erreurs différentes pouvant ou non être détectées par cette méthode. Roache présente aussi la méthode en détails dans [5] et a publié un livre sur le sujet (voir [6]).

L'intérêt principal de la MSM est qu'elle constitue une méthodologie systématique pour vérifier les calculs obtenus sur des problèmes de modélisation pour lesquels aucune solution analytique n'est connue. Ceci vient combler un besoin énorme, notamment pour les problèmes de mécanique des solides en grandes déformations. En utilisant la MSM, il sera désormais possible de vérifier qu'un logiciel donne le bon résultat. Cette technique a été jugée suffisamment fiable pour faire partie de la procédure standard de vérification de logiciels en mécanique des fluides (voir [7]) et en mécanique des solides (voir [8]). Il est intéressant de noter que l'« American Institute of Aeronautics and Astronautics » (AIAA) l'a incluse dans ses normes en 1998, alors que la « American Society of Mechanical Engineers » (ASME) l'a fait en 2006, soit beaucoup plus récemment. À notre connaissance, il n'existe aucune publication à ce jour qui fait usage de la MSM en mécanique des solides en $2 \mathrm{D}$ ou en $3 \mathrm{D}$, bien que l'on en trouve un exemple unidimensionnel dans le résumé 


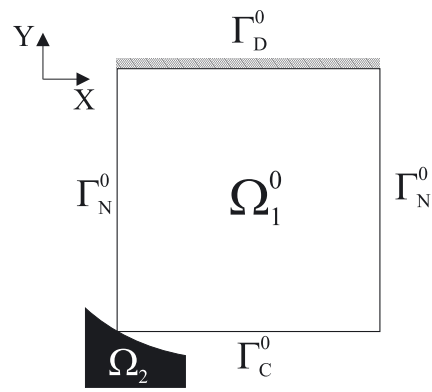

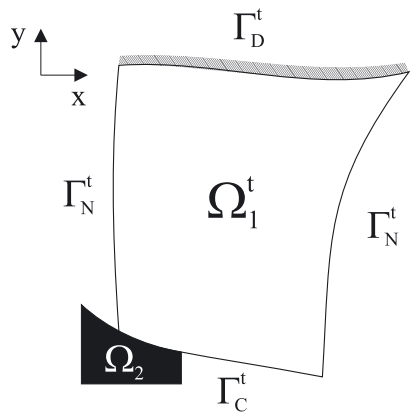

(a)

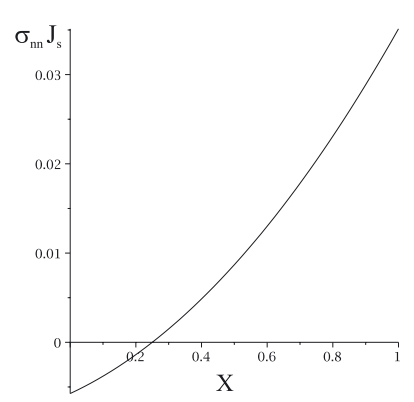

(b)

Fig. 1. (a) Domaine initial et déformé avec obstacle rigide, (b) contrainte normale $\left(\sigma_{\mathrm{nn}} J_{\mathrm{s}}\right)$ sur le bord $\Gamma_{\mathrm{C}}^{0}$.

du guide de l'ASME (voir Schwer [9]). Un article est en préparation sur le sujet (voir Chamberland et al. [10])

\section{Procédure de vérification de logiciels de contact unilatéral en grandes déformations}

Plusieurs développements informatiques délicats sont nécessaires pour la prise en compte des problèmes de contact dans un logiciel d'éléments-finis. L'un des plus importants est sans doute la mise en œuvre d'un opérateur de projection orthogonale d'un point $\left(\boldsymbol{x}_{1}\right)$ de la surface éligible au contact $\left(\Gamma_{\mathrm{C}}^{t}\right)$ sur l'objet rigide $\Omega_{2}$ (Fig. 1a). On obtient ainsi le point projeté $\left(\boldsymbol{x}_{2}\right)$ où l'on calcule la normale $\left(\boldsymbol{n}_{2}\left(\boldsymbol{x}_{2}\right)\right)$ pour ainsi définir la distance $(g=$ $\left.\left(\boldsymbol{x}_{1}-\boldsymbol{x}_{2}\right) \cdot \boldsymbol{n}_{2}\right)$ (le « gap » en anglais). On doit ensuite évaluer précisément une intégrale surfacique pour imposer la condition de non-pénétration. Le dernier élément important à mettre en place est l'extraction de la pression de contact résultant du calcul. Une fois ces éléments informatiques programmés, vient le moment de vérifier leur exactitude.

Pour le contact déformable-déformable, Taylor et Papadopoulos [11] proposent un test permettant de vérifier la bonne transmission des pressions de contact entre deux domaines déformables. Crisfield [12] reprend ce test et en propose une variante pour les problèmes de contact déformable-déformable avec une interface de contact courbe. Dans les deux cas, le problème proposé possède une solution analytique linéaire dont on peut extraire les composantes des contraintes de Cauchy. Toutefois, dans le cas des interfaces courbes, on ne montre pas comment calculer la contrainte normale $\left(\sigma_{\mathrm{nn}}\right)$ sur le bord en contact. Or, c'est précisément cette contrainte qui est la plus intéressante. De plus, la solution linéaire proposée est insuffisante pour tester des éléments quadratiques. Il serait en effet intéressant de pouvoir construire une solution quadratique et d'extraire les contraintes normales au bord de contact de manière à vérifier que les éléments quadratiques représentent exactement cette pression de contact.
Dans plusieurs travaux, on utilise le maillage le plus fin que l'on puisse se permettre pour obtenir une solution de référence. Il est alors possible d'étudier le taux de convergence sur une série de maillages plus grossiers. Il est aussi possible de comparer les résultats d'un logiciel de contact avec un logiciel reconnu. Par contre, comme le mentionne Roache [5], les éditeurs de ces logiciels « reconnus $»$ se gardent bien de publier leurs propres résultats de vérifications, et peut-être avec raison!

Nous considèrerons donc ici les problèmes de contact unilatéral en grandes déformations dans une formulation mixte en déplacements $\boldsymbol{u}$ et en pression de contact $\lambda$. Nous pourrons ainsi comparer, de manière équitable et libre d'erreur numérique, différents choix d'espaces de discrétisation $\boldsymbol{u}_{h}-\lambda_{h}$. Nous pourrons aussi effectuer une comparaison entre les éléments triangulaires et quadrangulaires, débat qui est toujours d'actualités en mécanique des solides. On pourra également comparer les solutions obtenues sur des maillages adaptés. La MSM permet de faire toutes ces comparaisons de façon très efficace.

Il est important de mentionner ici qu'il n'a pas été possible de trouver une procédure de vérification de calcul de contact en grandes déformations avec solution analytique pour les éléments de tout ordre d'approximation. La MSM, que nous allons maintenant décrire, permet de combler cette lacune par l'introduction de solutions analytiques d'ordre plus élevé que celles présentées dans [11,12] et El-Abbasi et Bathe [13].

Une procédure complète de vérification devrait inclure les items suivants :

1. Vérification que $\boldsymbol{u}_{h}$ (et en particulier sa restriction à la surface en contact $\left.\left.\boldsymbol{u}_{h}\right|_{\Gamma_{\mathrm{C}}^{0}}\right), \boldsymbol{\sigma}\left(\boldsymbol{u}_{h}\right)$ et $\lambda_{h}$ donnent la solution exacte pour une solution analytique comprise dans l'espace d'approximation de $\boldsymbol{u}_{h}-\lambda_{h}$;

2. Vérification du taux de convergence sur $\boldsymbol{u}_{h}$ (et $\left.\boldsymbol{u}_{h} \Gamma_{\mathrm{C}}^{0}\right), \boldsymbol{\sigma}\left(\boldsymbol{u}_{h}\right)$ et $\lambda_{h}$ pour une solution analytique qui n'est pas comprise dans l'espace d'approximation.

Pour les grandes déformations, une formulation lagrangienne totale en déplacement $\boldsymbol{u}$ sera utilisée. Le contact unilatéral sera traité par l'introduction d'un multiplicateur de Lagrange $\lambda$ et résolu par la méthode du gradient conjugué projeté (voir Tardieu et al. [14]). On note 
$\sigma_{\mathrm{nn}}^{-}$et $\sigma_{\mathrm{nn}}^{+}$les parties respectivement négative et positive de $\sigma_{\text {nn }}$. À convergence, le multiplicateur approchera $\sigma_{\mathrm{nn}}^{-} J_{\mathrm{s}}$, ce qui est lié à notre choix d'intégrer la contribution du jacobien surfacique $J_{\mathrm{s}}$ dans $\lambda_{h}$. Rappelons que $J_{\mathrm{s}}$ est le rapport $\mathrm{d} a / \mathrm{d} A$ entre les aires déformée et non déformée. Ceci évite de linéariser la variation de $J_{\mathrm{s}}$, ce qui introduirait un terme non symétrique dans la matrice tangente, comme dans le cas de l'imposition d'une pression suiveuse (voir Bonet et Wood [15]). Sous forme variationnelle, on a :

$$
\begin{gathered}
\int_{\Omega_{1}^{0}} \boldsymbol{\Pi}: \boldsymbol{\nabla}_{X} \boldsymbol{\phi}_{u} \mathrm{~d} V+\int_{\Gamma_{\mathrm{C}}^{0}} \lambda_{h} \boldsymbol{\phi}_{u} \cdot \boldsymbol{n}_{2} \mathrm{~d} A=\int_{\Omega_{1}^{0}} \boldsymbol{r}_{0} \cdot \boldsymbol{\phi}_{u} \mathrm{~d} V \\
+\int_{\Gamma_{\mathrm{N}}^{0}} \boldsymbol{h}_{0} \cdot \boldsymbol{\phi}_{u} \mathrm{~d} A+\int_{\Gamma_{\mathrm{C}}^{0}} \boldsymbol{k}_{0} \cdot \boldsymbol{\phi}_{u} \mathrm{~d} A \quad \forall \boldsymbol{\phi}_{u} \\
\int_{\Gamma_{\mathrm{C}}^{0}}\left(\phi_{\lambda}-\lambda_{h}\right) g \mathrm{~d} A \leq 0 \quad \forall \phi_{\lambda} \leq 0
\end{gathered}
$$

De manière équivalente, on a les équations habituelles du contact unilatéral :

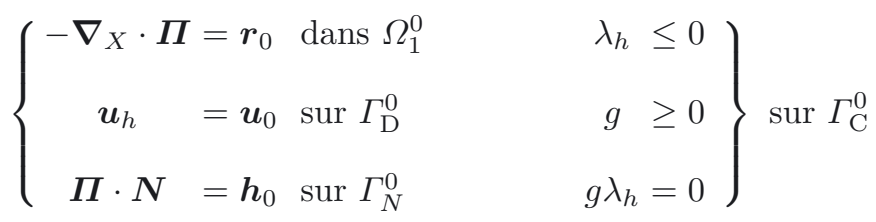

auquelles on ajoute un terme de bord faisant intervenir $\boldsymbol{k}_{0}=\boldsymbol{\Pi} \cdot \boldsymbol{N}+\lambda_{h} \boldsymbol{n}_{2}$ sur $\Gamma_{\mathrm{C}}^{0}$, de manière à préserver l'équilibre dans le système (1). En rappelant que sur $\Gamma_{\mathrm{C}}^{0}$, on a $\boldsymbol{n}_{2}=-\boldsymbol{n}$ pour la partie en contact et que $\lambda_{h}=\sigma_{\mathrm{nn}}^{-} J_{\mathrm{s}}$, on vérifie facilement que :

$\boldsymbol{k}_{0}=\boldsymbol{\Pi} \cdot \boldsymbol{N}+\lambda_{h} \boldsymbol{n}_{2}=\boldsymbol{\Pi} \cdot \boldsymbol{N}-\sigma_{\mathrm{nn}}^{-} \boldsymbol{n} J_{\mathrm{s}}=\left(\sigma_{\mathrm{nn}}^{+} \boldsymbol{n}+\boldsymbol{\sigma}_{\mathrm{t}}\right) J_{\mathrm{s}}$

et c'est la dernière expression de droite qui définit le terme supplémentaire en $\boldsymbol{k}_{0}$.

Le choix des espaces de discrétisation pour $\boldsymbol{u}_{h}$ et $\lambda_{h}$ est conditionné par une condition de type inf-sup (voir Bathe et Brezzi [16] et Coorevits et al. [17]). Plusieurs choix d'espaces de discrétisation satisfaisant cette condition seront présentés dans cette étude.

\section{Solution manufacturée pour le contact}

Nous présentons maintenant les principales étapes pour l'utilisation d'une solution manufacturée pour un problème de contact en grandes déformations.

1. Choisir un domaine de modélisation $\Omega_{1}^{0}$ ainsi qu'une solution manufacturée $\boldsymbol{u}$;

2. choisir la loi de comportement et les propriétés matérielles (le module de Young $E_{\mathrm{Y}}$ et le coefficient de Poisson $\nu$ pour un matériau hookéen);

3. calculer le gradient de déformation $\boldsymbol{F}$, le tenseur de Green-Lagrange $\boldsymbol{E}=\frac{1}{2}\left(\boldsymbol{F}^{\mathrm{t}} \cdot \boldsymbol{F}-\boldsymbol{I}\right)$ et les tenseurs de Piola-Kirchoff $\boldsymbol{S}$ et $\boldsymbol{\Pi}=\boldsymbol{F} \cdot \boldsymbol{S}$;

4. calculer la divergence du premier tenseur de PiolaKirchoff : $\boldsymbol{r}_{0}=-\nabla_{X} \cdot \boldsymbol{\Pi}$;
5. avec la normale $\boldsymbol{N}$ à la géométrie non déformée, calculer $\boldsymbol{h}_{0}=\boldsymbol{\Pi} \cdot \boldsymbol{N}$ pour l'imposition des conditions aux limites de type Neumann sur $\Gamma_{\mathrm{N}}^{0}$;

6. calculer le tenseur des contraintes de Cauchy $\boldsymbol{\sigma}=$ $\frac{1}{\operatorname{det}(F)} \boldsymbol{F} \cdot \boldsymbol{S} \cdot \boldsymbol{F}^{\top}$

7. calculer la normale à l'état déformé $\boldsymbol{n}=\frac{\boldsymbol{F}^{-\top} \cdot \boldsymbol{N}}{\left\|\boldsymbol{F}^{-\top} \cdot \boldsymbol{N}\right\|}$ et $J_{\mathrm{s}}=\operatorname{det}(\boldsymbol{F})\left\|\boldsymbol{F}^{-\top} \cdot \boldsymbol{N}\right\|$

8. calculer les contraintes normale $\sigma_{\mathrm{nn}}=(\boldsymbol{\sigma} \cdot \boldsymbol{n}) \cdot \boldsymbol{n}$ et tangentielle $\boldsymbol{\sigma}_{\mathrm{t}}=\boldsymbol{\sigma} \cdot \boldsymbol{n}-\sigma_{\mathrm{nn}} \boldsymbol{n}$;

9. choisir $\Gamma_{\mathrm{C}}^{0}$ tel que $\sigma_{\mathrm{nn}}<0$ sur une partie de celui-ci (si c'est impossible, alors recommencer à l'étape 1);

10. calculer $\boldsymbol{k}_{0}=\left(\sigma_{\mathrm{nn}}^{+} \boldsymbol{n}+\boldsymbol{\sigma}_{\mathrm{t}}\right) J_{\mathrm{s}}$ pour l'imposition des conditions aux limites sur $\Gamma_{\mathrm{C}}^{0}$;

11. créer l'obstacle rigide $\left(\Omega_{2}\right)$ correspondant à la déformée analytique de $\Gamma_{\mathrm{C}}^{0}$ où $\sigma_{\mathrm{nn}}<0$.

La figure 1a présente un exemple de problème assez complexe pour lequel une solution manufacturée a été construite. Le domaine initial est le carré $\left(\Omega_{1}^{0}=[0,1] \times\right.$ $[0,1])$. La solution manufacturée choisie est celle de l'équation (2) et une feuille de calcul (voir [18]) a été utilisée pour générer l'ensemble des variables nécessaires au calcul $\left(\boldsymbol{r}_{0}, \boldsymbol{h}_{0}, \boldsymbol{k}_{0}\right)$ et aux comparaisons $\left(\sigma_{\mathrm{nn}}, J_{\mathrm{s}}\right)$.

$$
\begin{array}{ll}
u_{X}=\frac{1}{50}\left(X-\frac{101}{4}+\frac{7}{2 \sqrt{51}}\right)^{2}+\frac{1}{50} Y^{5} X^{3} & E_{Y}=X+1 \\
u_{Y}=\frac{1}{50} Y^{4}\left(X-\frac{1}{2}\right)^{3}-\frac{1}{50} X & \nu=\frac{3}{10}
\end{array}
$$

Cette solution déforme le carré en une parabole sur la partie inférieure du carré $\left(\Gamma_{\mathrm{C}}^{0}\right)$. Sur cette frontière parabolique, la contrainte normale $\left(\sigma_{\mathrm{nn}}\right)$ est inférieure à 0 pour $X \in[0, a[$ (zone où il y aura contact) et plus grande que zéro pour $X \in] a, 1]$ avec $a=1 / 4$. La valeur analytique de $\left(\sigma_{\mathrm{nn}} J_{\mathrm{s}}\right)$ sur la frontière $\Gamma_{\mathrm{C}}^{0}$ est présentée à la figure $1 \mathrm{~b}$. L'obstacle rigide $\left(\Omega_{2}\right)$ a été représenté par un seul élément $P_{2}$ sur l'intervalle $X=[-1, a]$ et déformé par le déplacement donné par l'équation (2). Une série de maillages composés de quadrangles a été générée en divisant le domaine initial en $i$ subdivisions dans chaque direction, pour $i=[4,8,16,32,64,128,256,512]$. Les sommets des maillages résultants ont été aléatoirement perturbés d'un facteur $h / 10$ pour éviter les maillages trop réguliers provoquant parfois des taux de convergence trop optimistes. Tous les maillages de quadrangles ont été ensuite divisés en triangles.

\section{Résultats numériques}

Il est intéressant d'étudier la convergence de plusieurs variables. Nous nous concentrerons sur la convergence de $\lambda_{h}$ sur $\Gamma_{\mathrm{C}}^{0}$, car c'est la quantité d'intérêt pour un problème de contact. Bien entendu, l'erreur sur $\boldsymbol{u}_{h}$ peut être calculée en norme $H^{1}$ (Fig. 2). On constate que tout semble converger de manière optimale. Par contre, pour un problème de contact, il est crucial de regarder de plus près ce qui se passe sur la frontière de contact. Nous avons ainsi regardé la convergence du déplacement en norme $L^{2}$ sur le bord $\Gamma_{\mathrm{C}}^{0}$ (Fig. 3). La convergence ne semble plus 


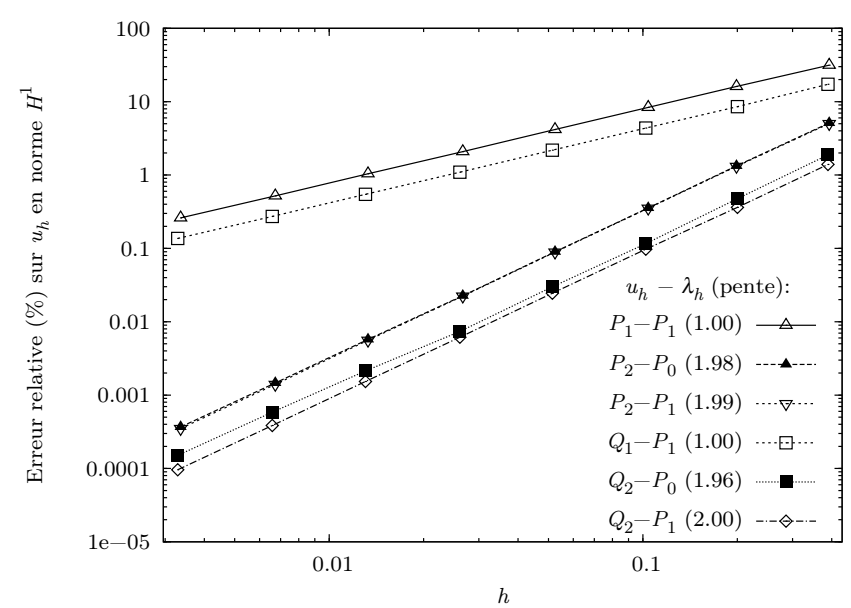

Fig. 2. Erreur relative en norme $H^{1}\left(\left\|\boldsymbol{u}-\boldsymbol{u}_{h}\right\|_{1, \Omega_{h}} /\|\boldsymbol{u}\|_{1, \Omega_{h}}\right)$ en fonction de la taille des éléments $(h)$.

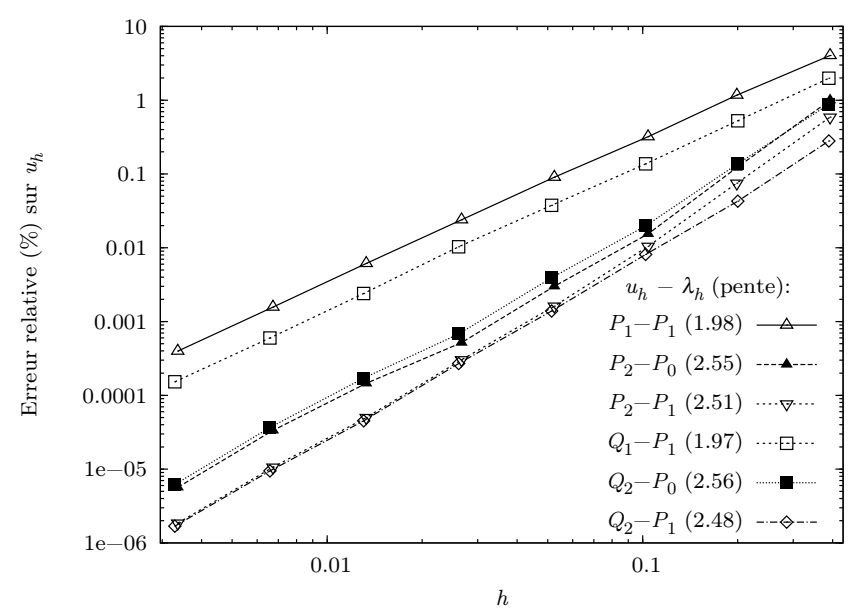

Fig. 3. Erreur relative en norme $L^{2}\left(\left\|\boldsymbol{u}-\boldsymbol{u}_{h}\right\|_{0, \Gamma_{\mathrm{C}}^{0}} /\|\boldsymbol{u}\|_{0, \Gamma_{\mathrm{C}}^{0}}\right)$ en fonction de la taille des éléments $(h)$.

optimale pour les éléments d'ordre 2 mais la théorie ne dit rien sur la convergence au bord dans cette norme. On sent toutefois la nécessité de regarder de plus près la convergence du multiplicateur.

On peut voir, à la figure $4 \mathrm{a}$, l'erreur relative en norme $L^{2}$ sur la pression de contact $\lambda_{h}$. On peut ainsi observer que $\lambda_{h}$ converge bien vers $\sigma_{\mathrm{nn}}^{-} J_{\mathrm{s}}$ (et non vers $\sigma_{\mathrm{nn}}^{-}$). On distingue aussi trois paires de courbes :

1. Les éléments les moins précis sont les $P_{2}-P_{0}$ et $Q_{2}-P_{0}$ pour lesquels la convergence est limitée à $O(h)$.

2. Les éléments $P_{1}-P_{1}$ et $Q_{1}-P_{1}$ ont aussi une convergence limitée à $O(h)$ mais sont deux fois plus précis que les précédents.

3. Les éléments $P_{2}-P_{1}$ et $Q_{2}-P_{1}$ sont limités dans leur convergence à $O\left(h^{1,54}\right)$, mais restent les plus précis de tous.

Dans ces résultats, on ne voit aucune différence marquée de précision entre les éléments triangulaires et quadrangulaires sauf en ce qui concerne l'erreur sur le déplacement pour le $P_{1}-P_{1}$ en comparaison avec le $Q_{1}-P_{1}$. Il est
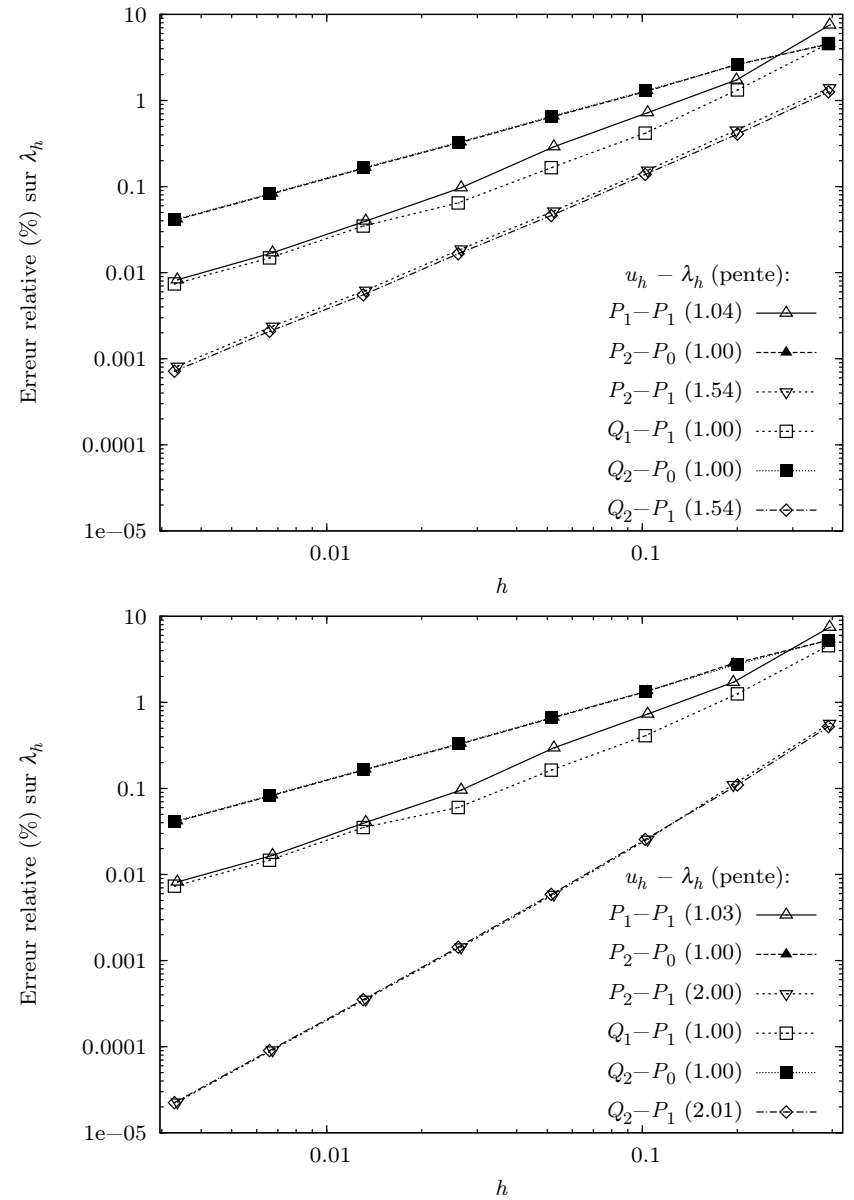

Fig. 4. Erreur relative sur $\lambda_{h}$ en norme $L^{2}$ sur $\Gamma_{\mathrm{C}}^{0}$ $\left(\left\|\sigma_{\mathrm{nn}}^{-} J_{\mathrm{S}}-\lambda_{h}\right\|_{0, \Gamma_{\mathrm{C}}^{0}} /\left\|\sigma_{\mathrm{nn}}^{-} J_{\mathrm{s}}\right\|_{0, \Gamma_{\mathrm{C}}^{0}}\right)$. (a) Maillages quelconques. (b) Maillages ayant un nœud en $X=1 / 4$.

toutefois bien connu que l'élément $P_{1}$ pour le déplacement est très pauvre et qu'il est à éviter.

Nous allons maintenant analyser le taux de convergence sous-optimal en $O\left(h^{1,54}\right)$ des éléments $P_{2}-P_{1}$ et $Q_{2}-P_{1}$. On peut proposer 2 explications : l'intégration numérique de $g$ ou l'absence de nœud sur la frontière de la zone de contact. Bien qu'une difficulté des problèmes de contact soit l'intégration exacte de $g$ (voir [13]), une analyse en cours nous a convaincu que le simple fait de ne pas avoir de nœud sur la frontière de la zone de contact est suffisant pour restreindre le taux de convergence de $\lambda_{h}$. Pour illustrer ce fait, nous présentons à la figure 4b les courbes de convergence obtenues sur des maillages ayant un nœud précisément à l'endroit où le changement de signe de $\sigma_{\text {nn }}$ se produit (soit à $X=1 / 4$ ). On retrouve alors immédiatement un taux de convergence $O\left(h^{2}\right)$ sur $\lambda_{h}$ pour les éléments $P_{2}-P_{1}$ et $Q_{2}-P_{1}$.

L'analyse théorique de la convergence et l'estimation a priori de l'erreur vont au-delà de la portée de ce travail. Hild et Laborde [19] présentent des résultats théoriques et numériques pour quelques espaces d'approximations. La convergence théorique est toutefois exprimée en norme $H^{-1 / 2}$ et il est difficile d'en donner l'équivalent en norme 
$L^{2}$. Nous pouvons cependant affirmer que l'on observe ici une convergence optimale de $\lambda_{h}$ dans la norme $L^{2}$ puisque l'interpolation de type $P_{1}$ assure au mieux un taux de convergence $O\left(h^{2}\right)$.

\section{Conclusions et perspectives}

L'originalité de ce travail réside dans l'utilisation de la MSM pour les problèmes de contact unilatéral en grandes déformations. La vérification de logiciel étant une partie incontournable de tout développement informatique, il est maintenant possible de le faire d'une manière plus complète qu'avec les méthodes classiques.

Nous avons montré comment on peut utiliser la MSM pour le contact unilatéral d'une manière très simple et facile à implémenter. Cela nous a permis de montrer l'importance de bien localiser la frontière de la zone de contact pour obtenir des taux de convergence optimaux. À cet effet, l'adaptation de maillage pourrait jouer un rôle primordial, ce qui fera l'objet d'une étude ultérieure.

Nous comptons également généraliser l'utilisation de la MSM aux problèmes de contact déformabledéformable, éventuellement avec frottement. Il serait possible de compléter le patch-test de contact proposé dans [11] et [12] avec des solutions analytiques $P_{2}$. Un autre intérêt de la MSM serait de comparer entre elles différentes méthodes de résolution des problèmes de contact (pénalisation, lagrangien augmenté, GCP). Il serait aussi possible de faire le même type de comparaisons pour les formulations mixtes incompressibles $\left(\boldsymbol{u}_{h}, p_{h}, \lambda_{h}\right)$, où la pression $(p)$ apparaît comme une inconnue supplémentaire.

\section{Références}

[1] C.J. Roy, Review of code and solution verification procedures for computational simulation, J. Comput. Phys. 205 (2005) 131-156

[2] S. Steinberg, P.J. Roache, Symbolic manipulation and computational fluid dynamics, J. Comput. Phys. 57 (1985) 251-284

[3] D. Pelletier, É. Turgeon, D. Lacasse, J. Borggaard, Adaptivity, Sensivity and Uncertainty: Towards Standards in CFD, In 39th AIAA Aerospace Sciences Meeting and Exibit, 2001

[4] K. Salari, P. Knupp, Code verification by the method of manufactured solutions, Sandia National Laboratories, 2000
[5] P.J. Roache, Code verification by the method of manufactured solutions, J. Fluids Eng. 124 (2002) 4-10

[6] P.J. Roache, Verification and validation in computational science and engineering, Hermosa, Albuquerque, 1998

[7] AIAA, Guide for the Verification and Validation of Computational Fluid Dynamics Simulations. Number G-077-1998. American Institute for Aeronautics and Astronautics, 1998

[8] ASME, Guide for Verification and Validation in Computational Solid Mechanics, Number V\&V 10-2006, American Society of Mechanics Engineers, 2006,

[9] L.E. Schwer, An overview of the PTC 60/V\&V 10 : guide for verification and validation in computational solid mechanics: Transmitted by l. e. schwer, chair ptc $60 /$ v\&v 10 Eng. Comput. 23 (2007) 245-252

[10] É. Chamberland, A. Fortin, M. Fortin, Comparison of the performance of some finite element discretizations for large deformation elasticity problems, Comput. Struct. 88 (2010) 664-673

[11] R.L. Taylor, P. Papadopoulos, in: Wriggers P., Wagner W. (eds.), Computational methods in nonlinear mechanics, chapter On a patch test for contact problems in two dimensions, Springer, 1991, pp. 690-702

[12] M.A. Crisfield, Re-visiting the contact patch test, Int. J. Numer. Meth. Eng. 48 (2000) 435-449

[13] N. El-Abbasi, K.-J. Bathe, Stability and patch test performance of contact discretizations and a new solution algorithm, Comput. Struct. 79 (2001) 1473-1486

[14] N. Tardieu, F. Youbissi, É. Chamberland, Un algorithme de gradient conjugué projeté préconditionné pour la résolution de problèmes unilatéraux, C. R. Mécanique 336 (2008) 840-845

[15] J. Bonet, D.R. Wood, Nonlinear continuum mechanics for finite element analysis, Cambridge University Press, Cambridge, 1997

[16] K.J. Bathe, F. Brezzi, Stability of finite element mixed interpolation for contact problems, Rend. Mat. Acc. Linceis. 9 (2001) 159-166

[17] P. Coorevits, P. Hild, K. Lhalouani, T. Sassi, Mixed finite element methods for unilateral problems: convergence analysis and numerical studies, Math. Comput. 71 (2002) 1-25

[18] É. Chamberland, Feuille Maple pour la méthode des solutions manufacturées en grandes déformations, http://www.giref .ulaval.ca/ ${ }^{\sim}$ ericc/mms

[19] P. Hild, P. Laborde, Quadratic finite element methods for unilateral contact problems. Appl. Numer. Math. 41 (2002) 401-421 